\title{
Cultura faz bem à saúde! A experiência do Circuito de Ocupação Cultural para a Saúde no Distrito Federal.
}

Culture is good for your health! The experience of the Cultural Occupation circuit for Health in the Federal District, Brazil.

\author{
¡ La Cultura es bueno para la salud! La experiencia del circuito cultural Ocupación \\ para la Salud en el Distrito Federal, Brasil.
}

\author{
Ana SCHRAMM \\ Felipe Medeiros PEREIRA ${ }^{2}$ \\ Ana Júlia TOMASINI ${ }^{3}$ \\ Nathália GAMEIRO ${ }^{4}$ \\ Luciana Sepúlveda KÖPTCKE ${ }^{5}$
}

RESUMO: O presente artigo analisa a experiência do Circuito de Ocupação Cultural pela Saúde, realizado, pelo Programa Educação Cultura e Saúde - Fundação Oswaldo Cruz, em parceria com as Secretarias de Saúde e de Cultura do Governo do Distrito Federal, com o apoio do Fundo de Apoio à Cultura (FAC-DF). A relação entre a Cultura e a Saúde constitui um importante empreendimento intersetorial, que ainda é emergente na busca pela promoção da saúde. Discutese, neste texto, as potencialidades das intervenções artísticas dentro dos espaços de saúde, a partir de uma perspectiva que busca valorizar a compreensão dos Determinantes Sociais de Saúde. Para tanto, foram sistematizadas as informações referentes às atividades realizadas pelo Circuito de Ocupação Cultural pela Saúde, de fevereiro de 2014 a maio de 2015, com a finalidade de levar para os equipamentos de saúde contrapartidas artísticas do Fundo de Apoio à Cultura. Dentre os dados disponíveis nos registros textuais das atividades, foram analisados quais foram os equipamentos de saúde mais atendidos pelo circuito, bem como o público alvo das atividades e as linguagens artísticas utilizadas. Discute-se, ainda, alguns fatores que facilitaram a implementação desta iniciativa. Palavras-Chave: Arte, Cultura, Promoção da Saúde, Ação Intersetorial, Humanização da Assistência.

\footnotetext{
ABSTRACT: The present article acknowledges the importance of Culture and Health collaboration as an important intersectoral endeavour, which is still emerging in the field of health promotion. It reports and analyses the Circuit of Cultural Occupation for Health Promotion Project, developed by the Education, Culture and Health Program - Fiocruz in partnership with

${ }^{1}$ Graduada em Artes Plásticas pela Universidade de Brasília,Brasil. E-mail:schramammana@gmail.com

${ }^{2}$ Graduado em Ciências Sociais pela Universidade de Brasília

${ }^{3}$ Graduada em Geografia pela Universidade de Brasília

${ }^{4}$ Graduada em Geografia pela Universidade de Brasília

${ }^{5} \mathrm{PhD}$ em Museologia pelo MNHN de Paris, Pesquisadora na Fiocruz Brasília. E-mail:lucianasepko@ gmail.com
} 
the Secretary of Culture and the Secretary of Health of the Federal District Government and the Supporting Culture Fund. The project addresses the potential of producing artistic interventions inside health institutions, aiming to enhance the understanding of culture and art as Social Determinants of Health. Therefore, we systematized the textual information referring to the activities implemented by the circuit from February 2014 to May 2015. The Circuit brings together artistic counterpart provided by the Supporting Culture Fund and the health institutions, in a way that the experience reported here has much to contribute to the discussion of the Cultural and Health interface in Brazil. Among the data analyzed, it is highlighted which kind of health institutions attended the circuit, as well as the public that participated in the activities and the artistic languages used. The article considers some elements that sustained the implementation of the Circuit of Cultural Occupation for Health Promotion.

Key words: Art, Culture, Health Promotion, Intersectoral Action, Humanization of Assistance.

RESUMEN: El presente artículo analiza la experiencia del Circuito de Ocupación Cultural por la Salud, realizado por el Programa de Educación Cultura y Salud - Fundación Oswaldo Cruz, en asociación con las Secretarias de Salud y de Cultura del Gobierno del Distrito Federal, con apoyo del Fondo de Apoyo a la Cultura. La relación entre la Cultura y la Salud constituye un importante emprendimiento intersectorial, que todavía es emergente en la búsqueda por la promoción de la salud. Se discute, en este texto, las potencialidades de las intervenciones artísticas dentro de los espacios de salud, a partir de una perspectiva que busca valorar la comprensión de los Determinantes Sociales de Salud. Para tanto, fueran sistematizadas las informaciones referentes a las actividades realizadas por el Circuito de Ocupación Cultural para la Salud, entre febrero de 2014 y mayo de 2015, con la finalidad de llevar para los espacios de salud contrapartidas artísticas del Fondo de Apoyo a la Cultura. Entre los datos disponibles en los registros textuales de las actividades, fueran analizados cuales fueran los espacios de salud más atendidos por el circuito, bien como los públicos privilegiados y los lenguajes artísticos utilizados. Se discute, aún, algunos factores que facilitaran la implementación de esta iniciativa.

Palabras clave: Arte, Cultura, Promoción de la Salud, Acción Intersectorial, Humanización de la Atención.

\section{INTRODUÇÃO}

Nos últimos 10 anos, observa-se no Brasil exercícios de aproximação formal entre a saúde e a cultura no campo das políticas públicas ${ }^{6}$ A busca pelo diálogo encontra significado a partir de, pelo menos, quatro processos em curso no país: a) fortalecimento da abordagem dos Determinantes Sociais da Saúde e da Promoção da Saúde (4); (5); (6); b) valorização de espaços mais democráticos de governança, participação e controle social; c) desenvolvimento de uma

\footnotetext{
${ }^{6}$ Em 2005 foi assinado um acordo de cooperação entre o MinC e a Fiocruz, tendo como objeto a Rede do Patrimônio histórico da Saúde e o Observatório de Museus e Centros Culturais, com gestão técnica da Casa de Oswaldo Cruz/ Fiocruz e do Departamento de Museus do IPHAN; Prêmio Cultura e Saúde 2008 Edital de Divulgação nº 3, de 6 de agosto de 2008; Prêmio Cultura e Saúde 2010 Edital de Divulgação nº 2, de 8 de março de 2010, para citar alguns.
} 
política cultural com foco na democracia cultural e nos direitos culturais, ampliando o entendimento da importância do setor para a cidadania; e, por fim, d) o investimento na construção de políticas intersetoriais para enfrentar os desafios da complexidade do combate às iniquidades em saúde. Estes processos têm como pano de fundo um modelo de desenvolvimento para o país que proponha articular crescimento econômico, sustentabilidade, inclusão social, enfrentamento às iniquidades entre pessoas e regiões e à pobreza.

Apesar de ser notória e crescente a produção acadêmica referente ao desenvolvimento de ações intersetoriais que têm um compromisso ético e político com o combate às desigualdades sociais existentes no país, principalmente no campo da Saúde Coletiva, tal bibliografia encontra-se demasiadamente focada na discussão e revisão conceitual da proposta intersetorial, e não no relato e avaliação dos empreendimentos na prática. Ainda incipientes no Brasil, as propostas intersetoriais são vistas como um experimento da gestão pública muitas vezes confundida como um artifício para tornar o processo de gestão mais eficiente, e não como consolidação de um projeto político comprometido em tornar o acesso a saúde de qualidade mais democrático no Brasil (1).

Mesmo diante desse quadro, podemos identificar que a Cultura vem paulatinamente conquistando seu espaço dentro dos equipamentos de saúde. Podemos citar como exemplos as intervenções em alas pediátricas que fazem uso da Palhaçaria (10), a utilização da arte-terapia no tratamento e na reabilitação social dos usuários da rede de Saúde Mental (12), bem como a proposta de modelos de articulação territorial entre instituições da cultura e da saúde (7).

Observando a necessidade de levar o empreendimento da intersetorialidade para além da discussão conceitual, a Rede Saúde e Cultura ${ }^{7}$ desenvolveu o Circuito de Ocupação Cultural para a Saúde (COCPS). Atuando como agente articulador, o Circuito reuniu representantes da Casa Civil, Secretaria de Saúde, Secretaria de Cultura e contou com o apoio das contrapartidas do Fundo de Apoio à Cultura (FAC) a fim de levar para dentro do equipamento de saúde atividades culturais de linguagens diversas, oferecidas pelos grupos culturais da sociedade civil. À Fiocruz coube identificar as demandas por atividades culturais dos equipamentos de saúde, ajudar a eleger a linguagem cultural adequada para cada espaço, promover o diálogo entre as partes e acompanhar e registrar todo o processo.

O presente artigo tem por objetivo analisar a utilização das linguagens artísticas em equipamentos de saúde, desenvolvidas nas duas primeiras etapas do Circuito de Ocupação Cultural para a Saúde. Dessa forma, foi possível identificar quais linguagens artísticas se fazem mais presentes e são mais adequadas para o diálogo com a esfera da saúde e, por conseguinte, com as especificidades de cada equipamento de saúde; o público alvo afetado pelo Circuito, particularmente identificando que tipo de linguagem artística é mais adequada para cada um deles. O número, a periodicidade e as datas, o tipo

\footnotetext{
${ }^{7}$ Termo de Cooperação № 01 de 21 de março de 2013 visa concretizar a articulação técnica entre competências da Fundação Oswaldo Cruz e do Ministério da Cultura, para execução do projeto Rede Saúde e Cultura:

"Fortalecimento das redes do Programa Cultura Viva com foco na diversidade cultural e sua articulação com a promoção da saúde" An-teriormente, foram firmados o Acordo de Cooperação 102/2010 e o Termo de Cooperação 134/2011 que promoveram o desenvolvimento de ações para implementação do projeto "Rede Saúde e Cultura para a promoção da qualidade de vida", em 2011/2012 
de atividade (contrapartida do FAC disponível) e a definição dos equipamentos onde as atividades ocorreriam foram pactuados em oficinas e reuniões anteriores ao início de cada etapa.

Participaram destas reuniões todos os parceiros envolvidos.

\section{METODOLOGIA}

Estudo exploratório e descritivo que articulou método quantitativo, qualitativo. A partir da análise de conteúdo do relatório final da primeira e da segunda etapa do projeto Circuito de Ocupação Cultural para Saúde $^{8}$, foi sistematizada parte dos dados a fim de analisar a utilização das linguagens artísticas em equipamentos de saúde. A análise de conteúdo é um método de pesquisa que entrou em amplo uso nos estudos da área de saúde nos últimos anos (11). Pode ser compreendido como um conjunto de técnicas de análise das comunicações visando obter, por procedimentos sistemáticos e objetivos de descrição do conteúdo das mensagens, a identificação dos temas e subtemas que permitam a compreensão e interpretação das mensagens analisadas. $\mathrm{O}$ método mostrou-se pertinente a este estudo, uma vez que tornou possível organizar as informações de forma a responder às questões de pesquisa (3). A análise partiu de macro temas definidos a priori: tipo de equipamento de saúde que participou do Circuito; público das atividades; natureza das atividades; linguagens artísticas utilizadas.

Foi realizada pesquisa bibliográfica exploratória utilizando como descritores termos como Humanização, Promoção da Saúde, Cultura e Saúde, Arte e Equipamentos de Saúde; na Biblioteca Virtual em Saúde - BSV, Scielo, Google Acadêmico e Arca.

O universo da pesquisa foram as 40 atividades culturais e educativas que ocorreram na primeira e segunda etapa do Circuito. Estas atividades foram descritas e interpretadas na sua relação com os equipamentos de saúde onde ocorreram, com a natureza das linguagens artísticas utilizadas em cada atividade, e com o público alvo para quem as atividades foram destinadas.

Após análise preliminar, as atividades foram organizadas nas seguintes categorias ou subtemas: $237 / /$

dança, palhaçaria, música, oficinas, apoio a eventos e teatro. Definiram-se também três categorias de público distintas: profissionais dos espaços de saúde, aqui compreendidos como os servidores e colaboradores destes espaços; usuários dos espaços de saúde aqui representados pelos pacientes de DST/AIDS, por crianças, mães nutrizes e grávidas de alto risco e por um público misto, reunindo tanto os funcionários como usuários e familiares.

\section{RESULTADOS}

A figura 1 apresenta a localização dos equipamentos que participaram do Circuito de Ocupação Cultural para a Saúde na primeira etapa, identificados pela cor vermelha. Foi o Hospital Regional da

\footnotetext{
${ }^{8} 8$ Relatório de Avaliação Preliminar das Duas Primeiras Etapas do Projeto Circuito de Ocupação Cultural para Saúde. PECS, Fiocruz, Brasília 2015 
Ceilândia - HRC; Centro de Atenção Psicossocial - CAPS II de Taguatinga; o Centro de Saúde No 1 de Planaltina; a Fiocruz Brasília e a Secretaria de Saúde do Governo do Distrito Federal - SES/GDF. Na segunda etapa, identificados pela cor azul, participaram o Hospital Psiquiátrico São Vicente de Paulo - HSVP; Hospital Materno Infantil de Brasília - HMIB; Centro de Atenção Psicossocial Infanto Juvenil do Plano Piloto - CAPSIJ; Centro de Referência em Atenção Psicossocial - CRAS de Sobradinho; Fiocruz Brasília. A Fiocruz é representada de forma diferenciada, por fazer parte das duas etapas. Os equipamentos participantes concentraram-se em ambos os lados do eixo leste - oeste, na periferia e no Plano Piloto.

Figura 1 - Mapa com localização dos equipamentos de saúde que receberam o Circuito de Ocupação Cultural para a Saúde.

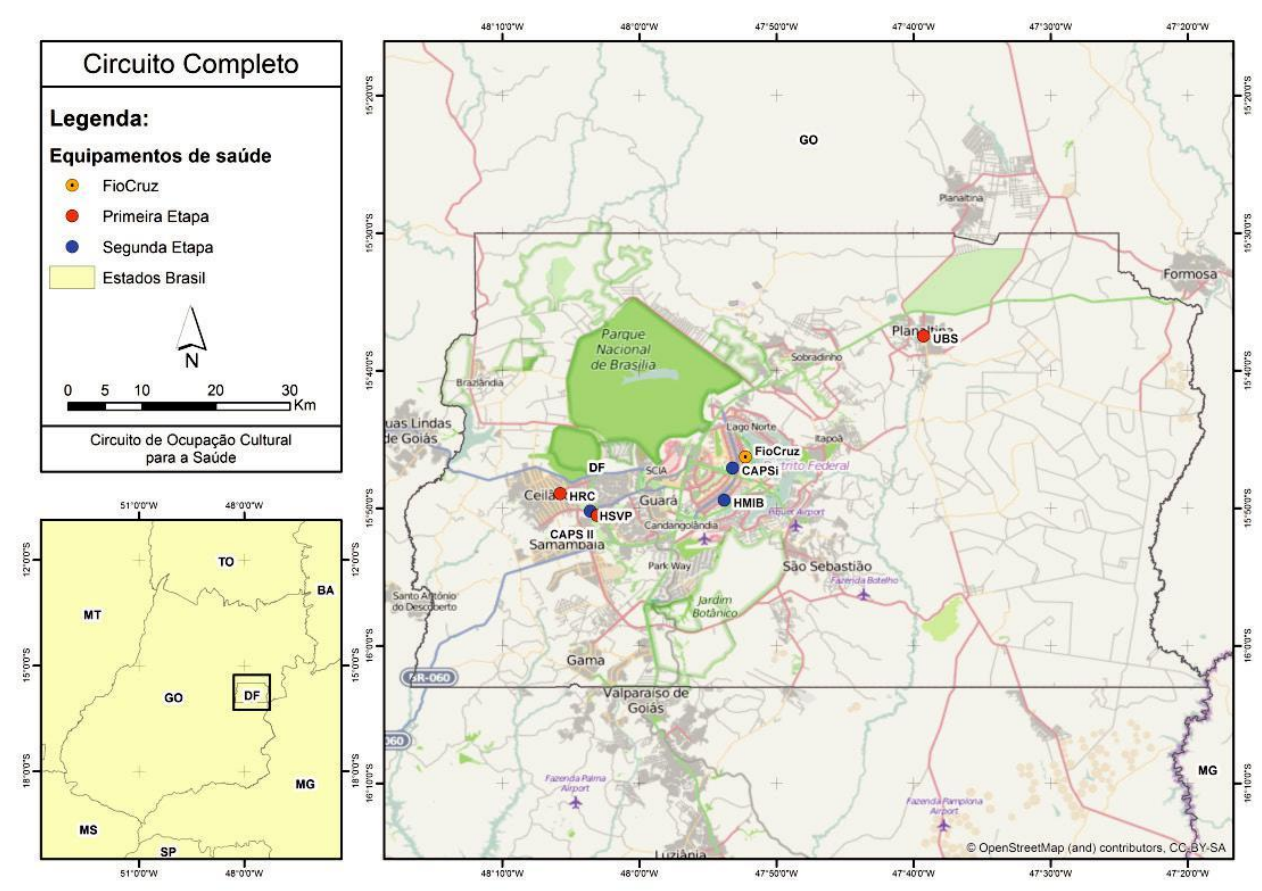

FONTE: Elaboração própria ARCGIS

O quadro 1 sintetiza informações acerca das atividades da $1^{\text {a }}$ etapa do Circuito. Foram descritos o tipo de equipamento, o local onde ocorreu a atividade, o público para quem foi realizada e a atividade, caraterizada por uma modalidade de linguagem artística. Nesta etapa, que ocorreu entre março de 2014 e julho de 2014, foram realizadas 18 (dezoito) diferentes atividades com diversos públicos. Duas atividades realizadas na SES, durante a Semana de Nacional de Humanização, foram incluídas a posteriori no planejamento do Circuito em resposta à demanda espontânea da SES. 
Quadro 1 - $1^{\text {a }}$ Etapa Circuito de Ocupação Cultural para Saúde

\begin{tabular}{|c|c|c|c|c|}
\hline EQUIPAMENTO & LOCAL & PÚBLICO & ATIVIDADE & TOTAL \\
\hline \multirow{5}{*}{$\begin{array}{l}\text { HOSPITAL REGIONAL } \\
\text { DA CEILÂNDIA }\end{array}$} & Refeitório & Profissionais da saúde & $\begin{array}{l}\text { Show musical - Diego } \\
\text { Galeno }\end{array}$ & \multirow{5}{*}{5} \\
\hline & Ala infantil & \begin{tabular}{|l|} 
Crianças internadas e \\
em atendimento
\end{tabular} & \begin{tabular}{|l|} 
Palhaçaria - Mandioca \\
Frita e Aipim \\
\end{tabular} & \\
\hline & Auditório & Mães nutrizes & $\begin{array}{l}\text { Oficina de cordel -Onã } \\
\text { Silva }\end{array}$ & \\
\hline & Brinquedoteca & Crianças internadas & $\begin{array}{l}\text { Espetáculo teatral - Cia } \\
\text { Cara de Palco }\end{array}$ & \\
\hline & Refeitório & Profissionais da saúde & Show musical - Arun & \\
\hline \multirow{4}{*}{ CAPS II TAGUATINGA } & Sala externa & Profissionais da saúde & $\begin{array}{l}\text { Oficina de teatro } \\
\text { Circo Íntimo } \\
\end{array}$ & \multirow{4}{*}{4} \\
\hline & Sala externa & Misto & $\begin{array}{l}\text { Oficina de cordel - Onã } \\
\text { Silva }\end{array}$ & \\
\hline & Sala externa & Misto & $\begin{array}{l}\text { Oficina de musica } \\
\text { Arun }\end{array}$ & \\
\hline & Feira da Torre de TV & Misto & $\begin{array}{ll}\text { Show } & \text { musical } \\
\text { Orquestra } & \text { Popular } \\
\text { Marafreboi } & \\
\end{array}$ & \\
\hline \multirow{3}{*}{$\begin{array}{l}\text { CENTRO DE SAÚDE Nº } 1 \\
\text { DE PLANALTINA }\end{array}$} & Sala de reunião & Profissionais da saúde & $\begin{array}{l}\text { Oficina de cordel } \\
\text { Onã Silva }\end{array}$ & \multirow{3}{*}{3} \\
\hline & Corredores & Misto & $\begin{array}{ll}\text { Palhaçaria } & - \text { Mostra } \\
\text { Zezito } & \\
\end{array}$ & \\
\hline & Ala DST/AIDS & Usuários & Show musical - Velt & \\
\hline \multirow{4}{*}{ FIOCRUZ } & $\begin{array}{l}\text { Espaço, Café, Ciência } \\
\text { e Cultura }\end{array}$ & Misto & $\begin{array}{l}\text { Show musical - Lucas } \\
\text { Soledade }\end{array}$ & \multirow{4}{*}{4} \\
\hline & Auditório externo & Misto & \begin{tabular}{|lr} 
Show musical \\
Geraldo Carvalho \\
\end{tabular} & \\
\hline & Auditório externo & Misto & $\begin{array}{l}\text { Oficina de dança } \\
\text { Educasalsa } \\
\end{array}$ & \\
\hline & Auditório externo & Misto & \begin{tabular}{|l|} 
Espetáculo musical \\
Ópera Don Giovanni \\
\end{tabular} & \\
\hline \multirow[b]{2}{*}{$\begin{array}{l}\text { SES } \\
\text { (Semana Nacional de } \\
\text { Humanização) }\end{array}$} & Auditório & Profissionais da saúde & Show musical - Arun & \multirow[b]{2}{*}{2} \\
\hline & Auditório & Profissionais da saúde & Show musical - Arun & \\
\hline TOTAL GERAL & & & & 18 \\
\hline
\end{tabular}

FONTE: Circuito de Ocupação Cultural pela Saúde 
O quadro 2 apresenta a $2^{\text {a }}$ etapa, especificando o equipamento, o local, o público e o tipo de atividade. Nesta etapa, que ocorreu entre agosto de 2014 e janeiro de 2015 foram realizadas 22 (vinte e duas) atividades. Também com grande variedade de categorias de atividades e públicos. Além dos 4 equipamentos de saúde escolhidos para esta etapa, o CRAS de Sobradinho recebeu duas atividades por indicação de parceiros estratégicos do projeto da Rede Saúde e Cultura. Ocorreram quatro atividades não planejadas nas oficinas iniciais. Uma na Fiocruz, outra no CAPSIJ e duas no HSVP.

Quadro 2 - 2a Etapa Circuito de Ocupação Cultural para Saúde

\begin{tabular}{|c|c|c|c|c|}
\hline EQUIPAMENTO & LOCAL & PÚBLICO & ATIVIDADE & TOTAL \\
\hline \multirow{4}{*}{$\begin{array}{l}\mathrm{H} \text { O S P I T A L } \\
\text { M A T E R N O } \\
\text { INFANTIL }\end{array}$} & Refeitório & \begin{tabular}{|l|}
$\begin{array}{l}\text { Profissionais } \\
\text { saúde }\end{array}$ \\
\end{tabular} & $\begin{array}{l}\text { Show musical - Grupo Vocal } \\
\text { Laugi }\end{array}$ & \multirow{4}{*}{4} \\
\hline & Sala da enfermagem & $\begin{array}{l}\text { Grávidas de alto } \\
\text { risco }\end{array}$ & Oficina de teatro & \\
\hline & Sala da enfermagem & $\begin{array}{l}\text { Grávidas de alto } \\
\text { risco }\end{array}$ & Oficina de teatro & \\
\hline & $\begin{array}{l}\text { E m e r g ê n c i a } \\
\text { ambulatório e ala da } \\
\text { internação }\end{array}$ & Misto & $\begin{array}{l}\text { Show musical gospel - Karlos } \\
\text { Vargas }\end{array}$ & \\
\hline \multirow{5}{*}{$\begin{array}{l}\text { CAPS IJ PLANO } \\
\text { PILOTO }\end{array}$} & Sala de aula & \begin{tabular}{|l|}
$\begin{array}{l}\text { Profissionais } \\
\text { saúde }\end{array}$ \\
\end{tabular} & Oficina de dança - Educasalsa & \multirow{5}{*}{5} \\
\hline & Sala de aula & \begin{tabular}{|l|}
$\begin{array}{l}\text { Profissionais } \\
\text { saúde }\end{array}$ \\
\end{tabular} & Oficina de dança popular e teatro & \\
\hline & Sala de aula & $\begin{array}{l}\text { Profissionais da } \\
\text { saúde }\end{array}$ & Oficina de dança popular e teatro & \\
\hline & Clube da Saúde & Misto & $\begin{array}{l}\text { Apresentação de dança - Black } \\
\text { Spin Break's }\end{array}$ & \\
\hline & Sala de aula & $\begin{array}{l}\text { Profissionais da } \\
\text { saúde }\end{array}$ & $\begin{array}{l}\text { Oficina de teatro - Tuka Villa } \\
\text { Lobos }\end{array}$ & \\
\hline \multirow{6}{*}{$\begin{array}{l}\text { H O S P I T A L } \\
\text { PSIQUIÁTRICO SÃO } \\
\text { VICENTE DE PAULA }\end{array}$} & Coreto & Misto & $\begin{array}{l}\text { Show musical - Nós Cegos na } \\
\text { Feira }\end{array}$ & \multirow{6}{*}{6} \\
\hline & Galpão & Misto & \begin{tabular}{ll|} 
Show musical - $\quad$ Grupo Vocal \\
Laugi
\end{tabular} & \\
\hline & Galpão & Misto & Espetáculo de dança - AMASSA & \\
\hline & Galpão & $\begin{array}{l}\text { Profissionais da } \\
\text { saúde }\end{array}$ & $\begin{array}{l}\text { Oficina de tango e interpretação } \\
\text { - Cia Contém Glúten }\end{array}$ & \\
\hline & Galpão & Misto & $\begin{array}{l}\text { Apresentação de dança - } \\
\text { Black Spin Break's }\end{array}$ & \\
\hline & Área externa & Misto & Picnik - Edição Pílula & \\
\hline
\end{tabular}




\begin{tabular}{|c|c|c|c|c|}
\hline \multirow{5}{*}{ FIOCRUZ } & \begin{tabular}{|l|} 
Espaço, Café, \\
Ciência e Cultura
\end{tabular} & Misto & $\begin{array}{ll}\text { Show musical }- & \text { Tambores } \\
\text { Urbanos }\end{array}$ & \multirow{5}{*}{5} \\
\hline & \begin{tabular}{|l|} 
Espaço, Café, \\
Ciência e Cultura
\end{tabular} & Misto & $\begin{array}{lll}\text { Show musical } & - & \text { Duo } \\
\text { Mandrágora } & & \\
\end{array}$ & \\
\hline & \begin{tabular}{|l|}
$\begin{array}{l}\text { Escola Fiocruz de } \\
\text { Governo }\end{array}$ \\
\end{tabular} & Misto & Oficina de objetos recicláveis & \\
\hline & Auditório externo & Misto & $\begin{array}{l}\text { Apresentação de dança - Black } \\
\text { Spin Break's }\end{array}$ & \\
\hline & Auditório externo & Misto & $\begin{array}{l}\text { Oficina de teatro - Tuka Villa } \\
\text { Lobos }\end{array}$ & \\
\hline $\begin{array}{l}\text { CRASSOBRADINHO } \\
\text { (Semana } \\
\text { Enfrentamento } \\
\text { Abuso e a Exploração }\end{array}$ & Auditório & Misto & Oficina de contação de historia & 2 \\
\hline $\begin{array}{l}\text { Adolescentes da Rede } \\
\text { Serrana) }\end{array}$ & Área externa & Crianças & Oficina de grafite & \\
\hline TOTAL & & & & 22 \\
\hline
\end{tabular}

FONTE: Circuito de Ocupação Cultural pela Saúde

As duas tabelas revelam as quarenta atividades e suas variáveis, como local, público e linguagem da atividade. A tabela 1 mostra que a Fiocruz e os Hospitais Regionais receberam o maior número de atividades.

Tabela 1 - Distribuição das atividades realizadas equipamento de nas duas etapas do Circuito

\begin{tabular}{|c|c|}
\hline Local & Número de atividades \\
\hline Hospital Regional & 9 \\
\hline Fiocruz & 9 \\
\hline CAPS & 8 \\
\hline Hospital Psiquiátrico & 6 \\
\hline Centro de Saúde & 3 \\
\hline Secretaria de Saúde & 2 \\
\hline CRAS & 2 \\
\hline Outros (espaços públicos) & 1 \\
\hline
\end{tabular}

FONTE: Circuito de Ocupação Cultural pela Saúde

Os Hospitais Regionais e a Fiocruz contaram com 22,5\% das atividades, seguidos pelos CAPS com 20\%. Apenas 2,5\% das atividades aconteceram em outros espaços.

Com o total de 40 atividades, o Circuito atingiu um público de aproximadamente 2100 pessoas. 
A primeira e a segunda etapa contaram com um público alvo variado devido à especificidade dos espaços de saúde e ao objetivo das atividades. A primeira etapa do Circuito atingiu cerca de 1000 pessoas e a segunda etapa cerca de 1100 pessoas.

Gráfico 2: Gráfico da Distribuição das Atividades por Categoria de Público. N = 40

\section{Distribuição das Atividades por Categoria de Público}

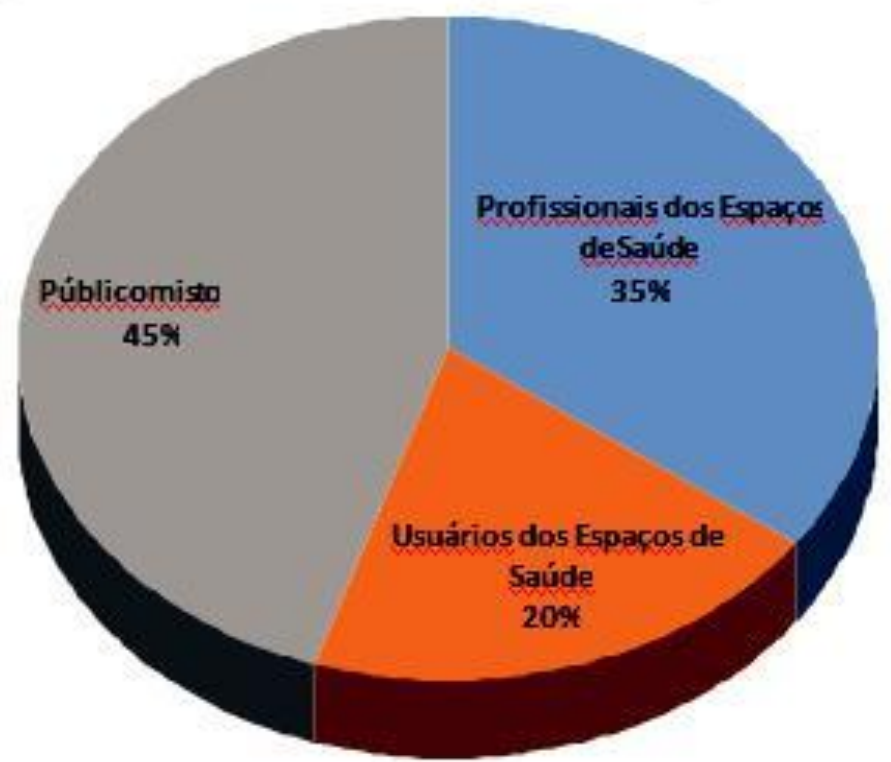

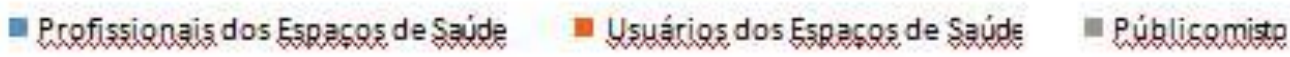

FONTE: Circuito de Ocupação Cultural pela Saúde

O gráfico mostra que a maioria das atividades do Circuito foi direcionada para o público misto, já que dentre as 40 atividades realizadas, 18 foram destinadas a esse público. Os profissionais dos equipamentos de saúde constituíram o segundo grupo mais atendido. No entanto, ao considerar que estes profissionais também foram beneficiados pelas atividades voltadas para o público misto, percebe-se que este segmento foi, globalmente, o mais frequente dentre os públicos do Circuito. 
Gráfico 3: Gráfico da Distribuição Linguagens Artísticas por número de incidências. $\mathrm{N}=$ 40

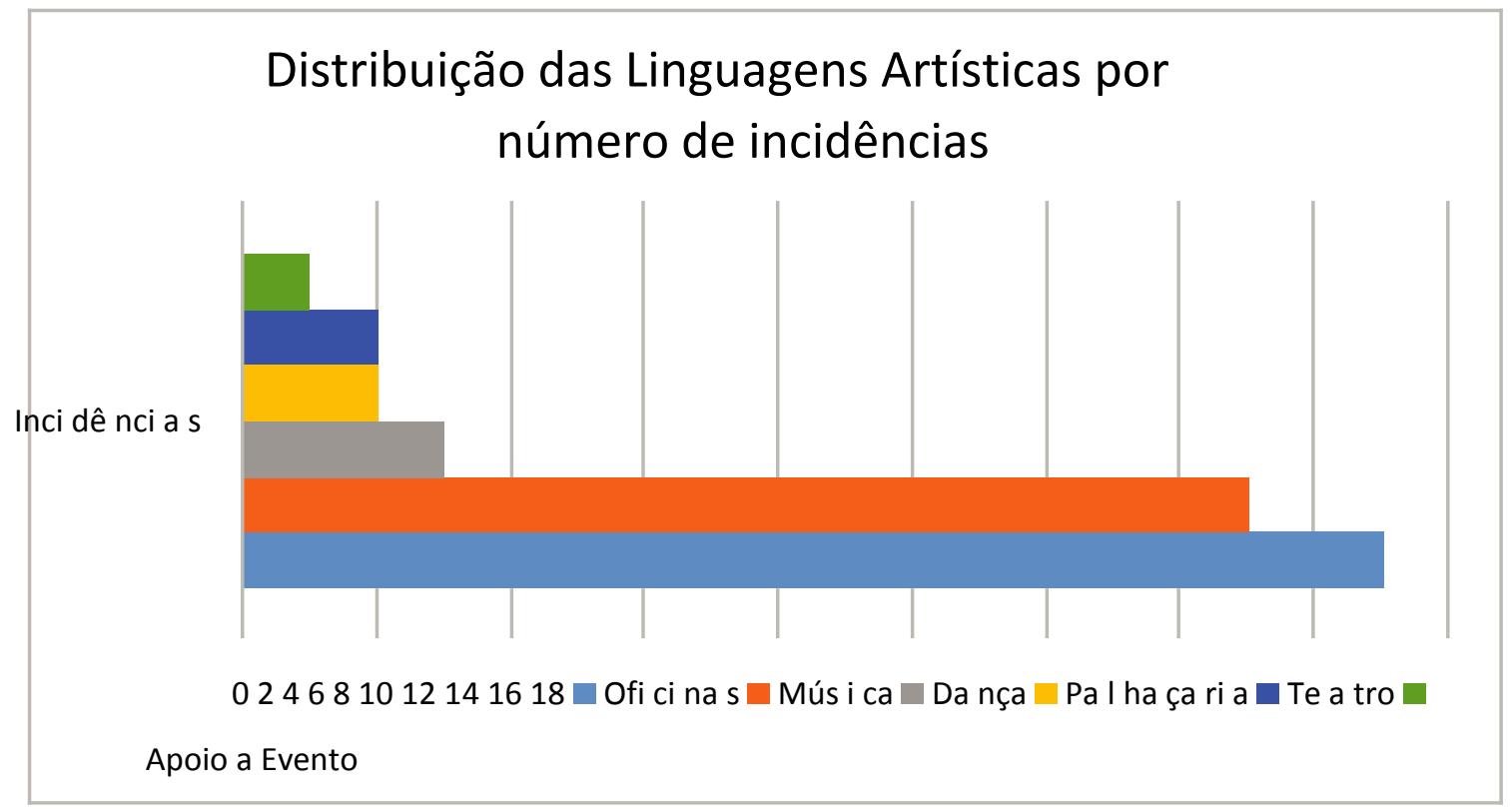

FONTE: Circuito de Ocupação Cultural pela Saúde

O gráfico indica as oficinas e atividades musicais como as linguagens mais frequentes dentro do Circuito. Dentre o total de 40 atividades, 42,5\% utilizaram a linguagem Oficina na sua apresentação, seguidas da Música que correspondeu a 37,5\% das apresentações realizadas.

Gráfico 4: A natureza das oficinas realizadas no Circuito de Ocupação Cultural para a Saúde.

\section{Natureza das Oficinas}

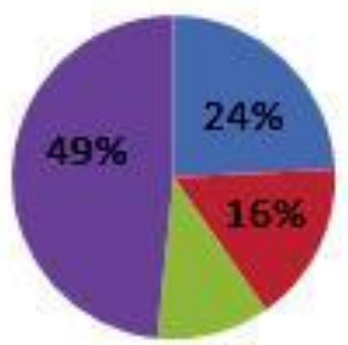

Cordel

Artes

Dança

Teatro

$11 \%$

FONTE: Circuito de Ocupação Cultural pela Saúde

O gráfico 4 ilustra a distribuição da linguagem artística utilizada nas Oficinas realizadas. Das 16 oficinas 03 foram de Leitura de Cordel, 02 oficinas de artes, 05 oficina de dança e 06 oficinas de teatro. A Oficina mais utilizada foi a de teatro.

As atividades do Circuito de Ocupação Cultural para a Saúde foram realizadas na maior parte das vezes em hospitais e nos CAPS. Voltadas, prioritariamente, para o público misto, seguido 
dos profissionais dos equipamentos da saúde, as oficinas foram o tipo de atividade mais frequente. Dentre as oficinas, o teatro foi a principal linguagem artística utilizada.

\section{DISCUSSÃO}

\section{Os equipamentos de saúde que mais participaram do Circuito}

A maior parte das atividades do COCPS ocorreu em hospitais e nos CAPS. A escolha das instituições de colhimento do Circuito foi determinada pelo gestor da SES, durante as reuniões preparatórias entre os parceiros da saúde, a Fiocruz e a SEC. Pode-se sugerir que tal escolha tenha refletido, por um lado, as prioridades e interesses da gestão em responder a demandas pontuais destes estabelecimentos.

Por outro lado, pode-se inferir que sejam estas as instituições do sistema de saúde com maior familiaridade em trabalhar com artistas ou agentes do campo cultural. Há quase três décadas projetos de palhaçaria em hospitais são práticas reconhecidas no Brasil como em todo o mundo. Tais práticas visam a humanização do acolhimento, a diminuição do estresse e promoção de melhor qualidade de vida e rápida recuperação, principalmente, nas alas de internação pediátrica (10).

Os CAPS e o HSVP fazem parte da rede de cuidado de saúde mental, uma área onde, tradicionalmente, se trabalha com a arte na perspectiva terapêutica, (12) bem como a partir da perspectiva da promoção de direitos, visando a inclusão social do sujeito em sofrimento psíquico e a construção de uma rede de atenção e serviços no território que se aproximam do espaço de vida, no cotidiano, onde oficinas artísticas disseminadas na malha urbana desempenham um importante papel de inclusão social e cultural (2). Nesta perspectiva, "las manifestaciones culturales associadas al movimento antimanicomial son un agente transformador de la actual ideologia globalizante y excluyente de nuestra sociedade" (8).

As atividades realizadas nos hospitais e nos CAPS nos levam a considera-los, pela sua finalidade e modo de funcionamento, ótimos lugares para receber as contrapartidas do FAC e atividades culturais de outros meios. Neste sentido, seria interessante propor ações continuadas e sustentáveis.

Estes equipamentos carecem de material para as oficinas e muitas vezes dependem de iniciativas de voluntários e de pesquisadores. Seria interessante também pensar em ações que abrangessem toda a rede de atenção psicossocial de forma integrada.

Uma só atividade ocorreu fora dos equipamentos de saúde. O show da Orquestra Popular Marafreboi, no espaço da Feira da Torre de TV, durante o Sarau Semeando Arte dos usuários da RAPS. A utilização de espaços públicos abertos para a realização de atividades culturais voltadas para pacientes, usuários e profissionais da saúde apontaram um enorme potencial a ser explorado, visto que "A medida que el modelo de atención deja de ser hospitalocéntrico [...] los usuarios y profisionales de la salud se mezclan com la comunidad [...] em los lugares frecuentados por el público de la ciudad" (2). 


\section{Como a atividade variou segundo o público?}

O Circuito enquanto projeto piloto se propôs a experimentar a realização de atividades culturais em diferentes equipamentos de saúde, compreendidos por hospitais regionais, centros de atenção psicossocial, unidades básicas de saúde e a Fiocruz. Cada tipo de equipamento de saúde aponta para potencialidades e desafios específicos, percebidos de acordo com a estrutura física e de pessoal, somados à mediação realizada pela equipe da Fiocruz e as atividades ofertadas pelo FAC.

Os dados revelam que a categoria de público mais atendido foi o misto para todos os equipamentos, apenas no HRC o público foi separado. Os profissionais da saúde participaram em 15\% a mais de atividades que os usuários dos espaços de saúde. Considerando que os profissionais participaram sozinhos de 35\% das atividades, cabe enfatizar o potencial desta parceria na promoção da saúde dos trabalhadores destes equipamentos. Pode-se inferir, ainda, com relação à maior frequência do público misto, que representasse uma opção capaz de assegurar maior número de pessoas participantes, ou ainda a facilidade em entrar na rotina do equipamento sem precisar mudar sua logística. Contudo, vale ressaltar que um dos objetivos principais do Circuito era a sensibilização dos profissionais da saúde e a humanização dos ambientes de cuidado, sendo isto, por tanto já esperado o direcionamento das atividades a este público.

O HRC na oficina de sensibilização e planejamento sugeriu atividades focadas para as mães nutrizes e servidoras que as acompanham, devido à existencia do trabalho educativo realizado no banco de leite; pelo menos duas ações voltadas para os servidores, que deveriam ocorrer na hora do almoço no refeitório, momento que reúne um bom número de servidores; uma para a pediatria, por ter uma brinquedoteca que já realiza atividades.

No HMIB detacou-se o público das grávidas de alto risco, que puderam receber duas oficinas de teatro. Estas foram indicadas durante a oficina de planejamento, por permanecerem muito tempo ociosas e desmotivadas, uma vez que precisam deixar suas familias e atividades devido as possíveis complicações da gestação. Já o HSVP apesar de ter planejado várias ações voltadas para os servidores, teve apenas uma para este público. Os servidores não conseguiam tempo para participarem e os usuários ficavam anciosos e curiosos para participarem das atividades, sendo assim optou-se por realiza-las para o público misto.

No caso do CAPS II de Taguatinga, como eles já ofereciam algumas atividades artísticas para os usuários realizadas pela própria equipe, assim como por voluntários, foi possível direcionar as ações do Circuito e encaixá-las na rotina do centro. Lá as atividades foram direcionadas para o público misto, com exceção da primeira atividade que foi apenas para os servidores. Já no CAPS IJ a maior número de atividades foram direcionadas para os servidores, uma vez que o fluxo de atendimento dos usuários é pequeno e espaçado. Sendo apenas a última realizada para o público misto, pois integrou um evento de final de ano do CAPSIJ. 
O Centro de Saúde $N^{\circ} 1$ de Planaltina, não pode participar da oficina de planejamento, mas na visita técnica foi sugerido realizar uma atividade voltada para os pacientes de DST/AIDS, uma para os pacientes da clínica médica e duas para os servidores. Foi possível realizar uma atividade exclusiva para os servidores, pois se aproveitou um dia de reunião de equipe, utilizando um tema de interesse comum, neste caso a enfermagem na leitura de cordel da Onã Silva. A segunda atividade, intervenção de palhaçaria Mostra Zezito, pode contar com um público misto, pois coincidiu com um dia especial de evento voltado para a terceira idade. Acredita-se que pelo perfil dos centros de saúde, o baixo quadro de funcionários e a própria rotina dificultem o direcionamento de atividades voltadas exclusivamente para os servidores.

O público da Fiocruz caracteriza-se por diferentes públicos: o público interno formado pelos servidores e colaboradores da instituição, o público externo formado pelos pesquisadores e estudantes das áreas de saúde que frequentam a instituição e pelo público externo geral que frequenta o campus universitário no qual a instituição está inserida. A comunidade da Fiocruz apresentou certa resistência para participar ativamente das atividades propostas. A participação no período do trabalho é intermitente. Entretanto, o fato da Fiocruz ter um coletivo responsável pela produção e planejamento das ações do Circuito e ter uma diversidade de espaços propícios para receberem as diferentes atividades ofertadas, facilitou a intervenção do Circuito.

\section{As diferentes Linguagens do Circuito}

O Circuito revelou que existem diversas possibilidades de utilização das linguagens artísticas nos equipamentos de saúde. Conforme a categoria de linguagem, público e espaço disponível pode-se realizar atividades com objetivos bem diversos. Algumas linguagens, como a música se adaptam em qualquer ambiente e público. Outras, como a dança e o teatro demandam uma estrutura específica. Já as oficinas possibilitam maior adesão e interação do público, apesar de serem para um número reduzido de pessoas.

As atividades realizadas pelo Circuito utilizaram diferentes linguagens artísticas, como palhaçaria, espetáculo de dança, espetáculo teatral, espetáculo musical, show musical e oficinas. As oficinas, por sua vez, se caracterizaram por uma grande diversidade de formatos e linguagens, como por exemplo, produção de literatura de cordel, danças populares, teatro, salsa, tango, contação de história, grafite e artesanato com materiais recicláveis. O Circuito apoiou, ainda, a realização do evento cultural PicniK Edição Pílula que não fazia parte das atividades oferecidas pelo FAC.

Os dados mostram que a categoria de linguagem oficina teve uma maior incidência, com 17 atividades. A preferência em utilizar contrapartidas de oficinas era uma diretiva do projeto. Tanto pelo seu caráter interativo, quanto pela sua maior duração e possibilidade de desdobramento.

O projeto previa que estas oficinas seriam direcionadas, preferencialmente para o público dos profissionais da saúde, no intuito de estimular atividades mais continuadas, e assim aumentar a 
efetividade das mudanças no ambiente de cuidado, na sensibilização dos artistas e dos profissionais da saúde.

Vale ressaltar, que a incidência de oficinas foi maior na segunda etapa do projeto, em decorrência do aprendizado adquirido da primeira etapa, que tornou evidente o potencial e sucesso da utilização deste tipo de atividade. As oficinas foram oferecidas não só para os profissionais da saúde, como para os usuários.

A linguagem música teve a segunda maior incidência com um total de 15 atividades. Isso deve-se tanto pelo fato de maior disponibilidade no banco de contrapartidas do FAC, como pela facilidade de encaixa-la em qualquer ambiente e público. A linguagem palhaçaria apesar da baixa incidência, apenas 2, representa uma linguagem que "dá certo" nos ambientes hospitalares. A intervenção com palhaços em equipamentos de saúde já é prática comum, a exemplo do trabalho dos Doutores da Alegria de SP, Doutoras Música e Riso, Grupo Risadinha e Anjapalhaços ambos de Brasília. Porém, o banco de contrapartidas do FAC não apresentava, naquele momento, outras atividades desta natureza para serem oferecidas no Circuito.

A ampla utilização de oficinas de teatro (49\%) pode ser compreendida tanto pela maior oferta destas atividades no banco de contrapartidas do FAC, quanto pela facilidade de adaptação da metodologia da oficina, a partir da negociação entre o artista e o equipamento de saúde. As oficinas de teatro, em geral, trabalham com linguagens híbridas, trazendo elementos da música, da poesia e da dança, o que permite essa adaptação de acordo com o público e com e ambiente.

\section{Importância da mediação entre culturas organizacionais diferentes}

Pode-se considerar que o Circuito de Ocupação Cultural para a Saúde, em suas duas primeiras etapas, atingiu os objetivos propostos de implementação, ultrapassando o número inicial de atividades previstas. Tal efeito sugere a receptividade dos equipamentos e a abertura dos artistas em colaborar com a iniciativa. Acredita-se que o retorno positivo, decorra, em parte, das ações de mobilização, articulação, produção e mediação junto aos artistas contemplados pelo FAC, às equipes da Secretaria de Saúde (SES), Secretaria de Cultura (SECULT) e aquelas dos equipamentos de saúde. Estas ações preparatórias e de acompanhamento ajudaram a caracterizar o perfil das atividades e o público alvo das ações do Ciruito. A articulação intersetorial provocada pelo COCPS enfrentou o desafio de construir o diálogo entre atores e serviços com pouca familiaridade em realizar atividades conjuntas. Uma das atribuições da Fiocruz no projeto foi mediar a ação, nas reuniões e oficinas. Este processo funcionou como um "dispositivo para a escuta qualificada e para o exercício do respeito à diferença e às diversidades na busca de possíveis interesses comuns, mesmo que temporários" (1). Ratifica-se -se a importância de prever espaços e situações para reconhecimento do parceiro, das diferenças e possibilidade de pactuação articulada. Tal arranjo não ocorre satisfatoriamente sem uma preparação, pois "como um processo coletivo e organizado a ação intersetorial não pode ser espontânea. Trata-se de uma ação deliberada que requer o respeito è diversidade e às particularidades 
de cada setor ou participante. Envolve espaços comunicativos, capacidae de negociação e inermediação de conflitos para a resolução do problema principal.” (9)

\section{CONCLUSÃO}

O presente artigo ao relatar a experiência do Circuito de Ocupação Cultural pela Saúde ensejou sistematizar os conhecimentos construídos no cotidiano da realização de uma iniciativa intersetorial. Apontou características específicas do processo de construção desta iniciativa, identificando as instituições com maior facilidade de acolhimento das iniciativas artístico-culturais, a prevalência de atividades híbridas, misturando linguagens artísticas, com alto grau de participação do público e flexibilidade como as oficinas, assim como os espetáculos musicais. Sugeriu o potencial destas atividades para colaborar com a Humanização dos serviços e equipamentos da Saúde bem como, com a promoção da saúde do trabalhador. Além disto, é importante ressaltar a relevância da construção de espaços de pactuação para que iniciativas intersetoriais como esta tenham maior chance de sucesso. Finalmente, espera-se colaborar com a implementação de um modelo de atenção que valorize o território e a construção de novas parcerias, onde os atores do campo cultural oferecem importante contribuição para a promoção da saúde.

\section{REFERÊNCIAS BIBLIOGRÁFICAS}

1. Akerman Marco, Franco de Sá Ronice, Moyses Simone, Rezende Regiane, Rocha Dais. Intersetorialidade? IntersetorialidadeS!. Ciênc. saúde coletiva

2. Amarante, P., Freitas, F., Rangel, P., M., Nabuco, E., El campo artístico-cultural en la reforma psiquiátrica brasileña: el paradigma identitário del reconocimiento, Salud Coletiva, 2013, 9(3).Lanus dic.

3. Bardin, Laurence. Análise de conteúdo.Lisboa:Edições 70, 2009.

4. Buss Paulo Marchiori, Pellegrini Filho Alberto. A saúde e seus determinantes sociais. Physis, 2007,17(1): 77-93 .

5. Buss P M., Pellegrini Filho A. Iniquidades em saúde no Brasil, nossa mais grave doença: comentários sobre o documento de referência e os trabalhos da Comissão Nacional sobre Determinantes Sociais da Saúde. Cad. Saúde Pública, 2006, 22(9): 2005-2008.

6. Buss P M, Carvalho A.I de. Desenvolvimento da promoção da saúde no Brasil nos últimos vinte anos (1988-2008). Ciênc. saúde coletiva . 2009, 14(6): 2305-2316. 
7. Camic P., Chatterjee H.J. Museums and art galleries as partners for public health interventions. Perspectives in Public Health. 2013;133:66-71

8. Corbella, L., Amarante, P., Cultura y Locura, Acción Cultural y Reforma Psiquiátrica, in Topia, un sitio de psicoanálisis, sociedad y cultura. Disponível em: http://www.topia.com.ar/ articulos/cultura-y-locura Acesso em 30.abr.2015.

9. Dias, M., S., de A., Parente, J.R.F., Vasconcelos, M.I.O., Dias, F.A.C, Intersetorialiade e Estratégia Saúde da Família: tudo ou nada a ver? Ciência e Saúde Coletiva, 19(11):4371-4382, 2014.

10. Françani G M, Zilioli D, Silva P R F, Sant'ana R P de M, Lima R A G de. Prescrição do dia: infusão de alegria. Utilizando a arte como instrumento na assistência à criança hospitalizada. Rev. Latino-Am. Enfermagem, 1998, 6(5): 27-33.

11. Hsieh, HF; Shannon, E. S. Three Approches to Qualitative Content Analysis. Qualitative Health Research, 15 (9), 2005.

12. Tavares C M. Oficina de arte: atuação terapêutica da enfermeira psiquiátrica. Rev. bras. enferm.,1997, 50(4): 569-576.

Artigo apresentado em 12-02-15

Artigo aprovado em 15-08-15

Artigo publicado no sistema em 22-12-15 